\title{
FAUNA DE MOSQUITOS ASOCIADA con Aedes aegypti EN GUADUAS, COLOMBIA, S.A.
}

\author{
Victor Alberto Olano', Milton E. Tinker
}

\begin{abstract}
Durante un estudio sobre la ecología del Aedes aegypti llevado a cabo en el área urbana de Guaduas, Colombia, se hallaron un total de siete especies de mosquitos que compartían hábitats con esta especie. Los criaderos en los cuales se encontró un mayor número de mosquitos asociados con el Aedes aegypti fueron los tanques bajos (albercas). Larvas de Toxorhynchites spp. se encontraron en tanques elevados. Se discute la importancia de estos hallazgos con relación a los aspectos de ecología y control del Aedes aegypti.
\end{abstract}

\section{INTRODUCCION}

Durante los años de 1978 y 1979 se llevó a cabo entre el Instituto Nacional de Salud (INS), el Servicio de Erradicación de la Malaria (SEM) y la Organización Panamericana de la Salud (OPS), un estudio en la zona urbana de Guaduas, Colombia, sobre la ecología del Aedes aegypti (Linnaeus, 1762) mosquito considerado en las Américas como transmisor de los virus del dengue y la fiebre hemorrágica del dengue, y como vector potencial de la fiebre amarilla urbana (1). Compartiendo hábitats con el Aedes aegypti se encontraron varias especies de mosquitos, algunas de las cuales se les considera transmisores de diferentes patologias y otras consideradas de importancia como agentes de control biológico.

En Colombia, muy pocos han sido los trabajos realizados para conocer la fauna de artrópodos presentes en áreas urbanas. Investigaciones realizadas en Cali, Valle, durante los años de 1974 y 1981 respectivamente, señalan a las especies Culex $(C)$ quinquefasciatus = fatigans (Say, 1823) y Culex nigripalpus (Theobald, 1901) como las especies de mosquitos más comunes en esta localidad $(2,3)$.

El propósito de esta publicación es informar sobre las especies de culicinos que se encontraron asociadas con el Aedes aegypti en Guaduas (Cundinamarca), como también ampliar el conocimiento sobre la fauna de mosquitos presentes en áreas urbanas.

\section{MATERIALES Y METODOS}

El área de estudio fue la localidad de Guaduas en el departamento de Cundinamarca. Su cabecera municipal está localizada a los $5^{\circ} 04^{\prime}$ de latitud norte y $74^{\circ} 36^{\prime}$ de longitud al oeste de Greenwich, con una área municipal de 783 km². Su altura aproximada sobre el nivel del mar es de 992 metros, temperatura media de $23^{\circ} \mathrm{C}$ y precipitación anual promedio de $1.135 \mathrm{~mm}$. Según la clasificación de Holdridge, esta zona corresponde a bosque seco tropical. El estudio fue llevado a cabo en el área urbana (600 casas) durante la época de Iluvia (20 de noviembre - 15 de diciembre/78, 16 de abril - 11 de mayo/79) y durante la época seca (16 de enero - 9 de febrero/79, 3 de julio - 23 de agosto/79).

\footnotetext{
1 Biólogo. Grupo de Entomologia, Instituto Nacional de Salud

2 Entomologo, OPS/OMS.
} 


\section{Encuesta larvaria}

Se inspeccionaron los criaderos potenciales (depósitos con agua) para Aedes aegypti y simultáneamente se recolectaban otras especies de culicinos. Los depósitos se clasificaron en 10 tipos: 1) tanques elevados, 2) tanques bajos (albercas), 3) barriles, 4) recipientes de barro, 5) latas, 6) botellas, 7) Ilantas, 8) árboles y plantas, 9) otros depósitos pequeños (floreros, bebederos de animales) y 10) miscelánea (canales de desagüe en los techos, chatarra, láminas, etc.).

\section{Recolección y manejo de las formas inmaduras}

Las larvas y pupas diferentes a Aedes aegypti, recolectadas en criaderos, se guardaban en cárpulas (tubos de vidrio o plástico de los utilizados para envasar anestesia en dentistería) con alcohol al $70 \%$, las cuales se rotulaban indicando el tipo de depósito, lugar, fecha y recolector. Este material se transportaba al Laboratorio de Entomología del INS en Bogotá para su identificación. Para la identificación de las formas inmaduras encontradas se usaron las claves taxonómicas de Forattini y Lane (4 - 7).

\section{RESULTADOS}

Un total de 7 especies de culicinos diferentes a Aedes aegypti se encontraron e identificaron en el área urbana de Guaduas. Además, se recolectaron algunas especies no identificadas de los géneros Psorophora y Toxorhynchites (Tabla 1). La especie más abundante encontrada después del Aedes aegypti fue el mosquito Culex (C) quinquefasciatus; los tanques bajos (albercas) y las llantas figuraron entre los criaderos en los cuales esta especie se halló asociada al Aedes aegypti. El mosquito Aedes fluviatilis (Lutz,1904) se encontró desarrollándose en barriles y tanques bajos (albercas). Las especies Culex nigripalpus y Culex corniger (Theobald, 1903) se encontraron también en albercas, asociadas al Aedes aegypti. Larvas del género Toxorhynchites se hallaron en tanques elevados; formas inmaduras del mosquito Limatus durhami (Theobald, 1901), se recolectaron en Ilantas, las cuales son también un criadero muy importante para Aedes aegypti.

TABLA 1. Especies de mosquitos recolectados en el área urbana de Guaduas, (Cundinamarca)

\begin{tabular}{|c|c|c|c|c|c|c|c|c|c|c|}
\hline \multirow[t]{2}{*}{ ESPECIE } & \multicolumn{10}{|c|}{ TIPO DE DEPOSITO } \\
\hline & $\begin{array}{l}\mathbb{N} \\
\stackrel{\mathbb{T}}{ \pm} \\
\frac{\mathbb{N}}{J}\end{array}$ & 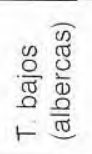 & 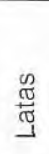 & 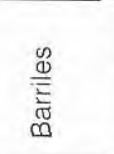 & 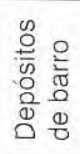 & 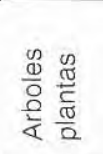 & $\begin{array}{l}\frac{\mathscr{D}}{\mathbb{W}} \\
\overline{\bar{\Phi}} \\
\stackrel{0}{0} \\
\infty\end{array}$ & 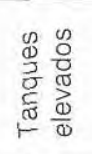 & 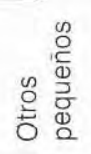 & 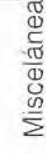 \\
\hline Aedes $(O)$ fluviatilis & - & + & - & + & - & - & - & - & - & - \\
\hline \multicolumn{11}{|l|}{ Culex (C) quinque- } \\
\hline fasciatus & + & + & & - & - & - & - & - & - & - \\
\hline Culex $(C)$ corniger & - & + & - & - & - & - & - & - & - & - \\
\hline Culex (C) coronator & - & + & - & - & - & - & - & - & - & - \\
\hline Culex (C) nigripalpus & - & + & - & - & - & - & - & - & - & - \\
\hline Culex (L) allostigma & - & - & - & + & - & - & - & - & - & - \\
\hline Limatus durhami & + & - & - & - & - & - & - & - & - & - \\
\hline Psorophora spp. & - & - & - & + & - & - & - & - & - & - \\
\hline Toxorhynchites spp. & - & - & - & - & - & - & - & + & - & - \\
\hline
\end{tabular}




\section{DISCUSION}

Se encontraron en los depósitos formas inmaduras de tres especies que son muy similares macroscópicamente al Aedes aegypti y que pueden llevar a errores de identificación. Estas especies son: Aedes fluviatilis, Limatus durhami y Culex corniger. La confirmación taxonómica de estas especies se debe realizar bajo un estereomicroscopio. La especie Aedes fluviatilis ha sido hallada asociada al Aedes aegypti en criaderos artificiales en Brasil y a partir de ella se ha aislado el virus de fiebre amarilla en ese mismo país (4). Al mosquito Culex corniger se le ha encontrado en Cuba en depósitos artificiales (latas) los cuales acumulan agua de lluvia $(8,9)$. En Panamá y Brasil en el ambiente doméstico se reportó la especie Limatus durhami $(4,10)$. Con relación a la especie Culex (C) quinquefasciatus se le considera vectora de filariasis y de algunas encefalitis $(4,11)$. En Colombia, esta especie aun cuando no se le considera transmisora de estas patologías, constituye molestia sanitaria enáreas urbanas. A losmosquitos Culex coronator (Dyar and Knab, 1925) y Culex nigripalpus se les ha encontrado naturalmente infectados con el virus de San Luis; la última de las especies mencionadas ha sido hallada infectada en Trinidad con el virus de la encefalitis del Este y en Panamá con el virus llheus $(4,11)$. Estas dos especies se han encontrado en el área urbana de la ciudad de Cali (3); criaderos urbanos de Culex nigripalpus se han encontrado en Cuba $(8,12)$. Por otro lado la especie Culex (Lutzia) allostigma (Howard, Dyar and Knab, 1915) y especies del género Toxorhynchites se les considera depredadores de larvas del Aedes aegypti $(6,13,14)$. Formas inmaduras de la especie Toxorhynchites portoricensis se han encontrado en el municipio de Guiness en Cuba (12).

\section{SUMMARY}

Seven different species of mosquitoes were found sharing the natural habitat of Aedes aegypti, during a study of the ecology of this vector in Guaduas, Colombia. Low tanks used for laundry (albercas) were the breeding places where Aedes aegyptiexhibited the greatest degree of association with other species. Larvae of Toxorhynchites $\mathrm{spp}$. were found in elevated water reservoirs. The relevance of these findings for the ecology and control of Aedes aegypti is discussed.

\section{AGRADECIMIENTOS}

Se agradece el apoyo recibido por parte del Grupo de Entomología del INS y de los funcionarios de la Zona XIII de Honda del Servicio de Erradicación de la Malaria (SEM). De manera especial se agradece a las autoridades y a la población de Guaduas por su colaboración y ayuda para llevar a cabo este estudio. A María Herly López por la transcripción del presente artículo.

\section{REFERENCIAS}

1. Tinker ME, Olano VA. Ecologia del Aedes aegyptien un pueblo de Colombia. Biomedica 1993: 13:5-14.

2. Barreto P. Sobre la presencia de algunos artrópodos en el área urbana de la ciudad de Cali. Acta Médica del Valle $1974 ; 5: 122$.

3. Fajardo P, Arias A. Mosquitos comunes en Cali (Colombia). Colombia Médica 1981; 12: 20.

4. Forattini OP. Entomologia Médica. Sao Paulo: Editora da Universidade de Sao Paulo; 1965: Vol. II.

5. Forattini OP. Entomologia Médica. Sao Paulo: Editora da Universidade de Sao Paulo;1965: Vol III.

6. Lane J. Neotropical Culicidae. Sao Paulo: Editora da Universidade de Sao Paulo, 1953: Vol. I.

7. Lane J. Neotropical Culicidae. Sao Paulo: Editora da Universidade de Sao Paulo; 1953: Vol. II.

8. Pérez Diaz R, Fuentes González O. Análisis del sistema de vigilancia por larvitrampas en el municipio Mariel (1984-1987). Rev Cubana Med Trop 1990; 42: 254.

9. García Avila I. Fauna cubana de mosquitos y sus criaderos típicos. Academia de Ciencias de Cuba. 1977: 46.

10. Lourenco de Oliveira R, Heyden R, Da Silva TF. Various aspects of the ecology of mosquitoes (Diptera: Culicidae) from a plains area (Calabria forms) in Jacarepagua Río de Janeiro. V. Breeding grounds. Mem Inst Oswaldo Cruz 1986; 81: 265. 
11. Organización Panamericana de la Salud. El control de las enfermedades transmisibles en el hombre. Publicación Científica No. 442, 1983.

12. Bisset JA, Marquetti MC, González B, et al. La abundancia larval de mosquitos urbanos durante la campaña de erradicación del Aedes aegypti (Linnaeus, 1762) y del dengue en Cuba (1981-82). Rev Cubana Med Trop 1985; 37: 161.
13. Focks DA, Sackett SR, Bailey DL. Field experiments on the control of Aedes aegypti and Culex quinquefasciatus by Toxorhynchites rutilus rutilus (Diptera: Culicidae). J Med Entomol 1982; 19: 336.

14. Focks DA. The integrated use of $T x$. amboinensis and ground-level ULV insecticide application to suppress Ae. aegypti. J Med Entomol 1986; 23: 513. 